\title{
Psychotropic drug prescribing in a Nigerian psychiatric hospital
}

\section{Imafidon O. Agbonile ${ }^{1}$ and Oluwole Famuyiwa²}

\author{
${ }^{1}$ Consultant Psychiatrist, Psychiatric Hospital, Uselu, PMB 1102, Benin City, Edo State, Nigeria \\ 2Professor of Psychiatry, Lagos University Teaching Hospital, Nigeria, email fam2005lag@yahoo.com
}

$P^{s}$ sychopharmacotherapy dominates the therapeutic arsenal of psychiatrists and, not surprisingly, psychotropic drugs are widely consumed in psychiatric practice. The pattern of prescribing of these drugs needs to be appraised in terms of 'rational drug use', which may be defined as 'the use of the least number of drugs to obtain the best possible effects in the shortest possible time and at a reasonable cost' (Gross, 1981).

Observations on prescribing practice can be the basis of useful biomedical research (Barbul \& Tansella, 2005) and are a form of self-audit for hospitals (Edwards \& Kumar, 1984). Several studies have noted variations in prescribing patterns in different settings (Xiang et al, 2007b). These variations (which may be related to poverty, lack of community resources and treatment adherence) and conformity with rational drug use in Nigeria were investigated in this study.

\section{Methods}

We enquired into the pattern of prescribing psychotropic drugs at the Uselu psychiatric hospital, Benin City, Nigeria, a 250-bed institution run by the federal government which serves an estimated population of 13 million.

We randomly chose a census day in March 2007 and extracted prescribing data on all 143 in-patients, none of whom had a physical disorder that might have influenced the psychotropic prescription. Five case files were excluded because the record was inadequate. The data colleced were key demographic factors, type of medication, frequency of use and administration schedules of prescribed drugs, their mean daily doses, combinations, use of anticholinergics, 'when required' (p.r.n.) orders and monthly costs of the drugs to the patients.

The data were processed using SPSS version 10 and a $5.0 \%$ significance level was used for comparison of frequencies using chi-square statistics.

\section{Results}

Prescriptions of antipsychotics, anxiolytics, anticholinergics, antidepressants and mood stabilisers varied significantly across some demographic variables: gender, Kruskal-Wallis value $(\mathrm{KW})=13.76$, d.f. $=4, P<0.001$; marital status, $\mathrm{KW}=20.23$, d.f. $=16, \quad P<0.005$; age (10-year groupings), $K W=19.73$, d.f. $=16, P<0.05$; and employment, $\mathrm{KW}=21.51$, d.f. $=8, P<0.05$. These distributions reflect the characteristics of patients seen at the hospital rather than the specifics of prescribing practice there.

The survey revealed a high frequency of prescribing of conventional antipsychotics to all patients with functional psychotic disorders: $51.1 \%$ of these patients were prescribed haloperidol, $45.5 \%$ chlorpromazine, 38.5\% trifluoperazine, $10.5 \%$ fluphenazine decanoate. There was less frequent prescribing of the atypical (second-generation) antipsychotics: $4.9 \%$ risperidone and $1.4 \%$ olanzapine.

Of the 15 patients with a depressive disorder, 10 were prescribed amitriptyline (a tricyclic antidepressant), three sertraline, one paroxetine and one fluoxetine. Regarding the mood stabilisers, for bipolar affective disorder, 15 patients were prescribed carbamazepine and 4 sodium valproate. One patient received a high dose $(450 \mathrm{mg})$ of thioridazine.

The mean daily doses of all drugs prescribed were within acceptable therapeutic ranges for the respective diagnoses; however, there was a remarkable consistency in the p.r.n. duo of intramuscular chlorpromazine $150 \mathrm{mg}$ and intravenous diazepam $30 \mathrm{mg}$ for rapid tranquillisation of severely agitated patients. Generally we found no anomalies in the matching of drug types with diagnostic groups.

Polypharmacy was noted in $92.3 \%$ of all prescriptions, with $62.2 \%$ of patients being on antipsychotic/anticholinergic combinations (Table 1). The commonest adjunctive drug was an anticholinergic agent, benzhexol, which was noted in the prescriptions of $82.6 \%$ of patients placed on regular antipsychotics and was administered once or twice daily. The frequency of p.r.n. prescribing was $57.7 \%$ and was for patients with non-affective psychotic disorders, either for sedation or to control extrapyramidal reactions. The other major finding was that $36.4 \%$ of prescriptions were to be administered twice and $6.8 \%$ thrice daily.

The monthly costs of the typical antipsychotics prescribed varied between US $\$ 2.44$ for fluphenazine decanoate to US\$12.0 for thioridazine, while the generally preferred atypical antipsychotics cost much more: risperidone US\$17.00 and olanzapine US\$36.70. For the treatment of depression, the monthly cost of the most frequently prescribed antidepressant, amitriptyline, was US\$5.00, which is much cheaper than the selective serotonin reuptake inhibitors: sertraline US\$18.0, paroxetine US\$60.90 and fluoxetine US\$65.00.

\section{Discussion}

The most discernible practice was polypharmacy, as has previously been reported in Nigeria (Famuyiwa, 1988) and 


\begin{tabular}{|c|c|c|}
\hline Drug combinations & Number of prescriptions & Percentage of total \\
\hline Antipsychotic alone & 11 & 7.7 \\
\hline Antipsychotic + anticholinergic & 89 & 62.2 \\
\hline Antipsychotic + anxiolytic & 3 & 2.1 \\
\hline Antipsychotic + anxiolytic + anticholinergic & 5 & 3.5 \\
\hline Antipsychotic + antidepressant & 5 & 3.5 \\
\hline Anxiolytic + antidepressant & 1 & 0.7 \\
\hline Antipsychotic + mood stabiliser & 5 & 3.5 \\
\hline Antipsychotic + mood stabiliser + anticholinergic & 13 & 9.1 \\
\hline Antipsychotic + antidepressant + anticholinergic & 9 & 6.3 \\
\hline Antipsychotic + mood stabiliser + anticholinergic + anxiolytic & 1 & 0.7 \\
\hline Anxiolytic alone & 1 & 0.7 \\
\hline Total & 143 & 100.0 \\
\hline
\end{tabular}

in numerous other studies (see Cookson et al, 2002) but its rationale has been questioned, because of its high risk of dangerous interactions and the burden it imposes on both nurses and the patient, as well as carers. However, polypharmacy may be justified for some patients who are unresponsive to single-drug treatments, provided close monitoring for undesirable effects or toxicity is ensured (Broekema et al, 2007). Combinations of two or more drugs should generally be avoided, but if a combination is judged to be necessary then the drugs should belong to different chemical groups (Cookson et al, 2002).

The liberal use of anticholinergic drugs was probably due to the high rate of prescribing typical antipsychotics, as also reported in several other studies (Xiang et al, 2007a). Although anticholinergic drugs are useful in controlling extrapyramidal reactions, they may be misused by some patients, as they can give the experience of euphoria, and they can cause cognitive impairment (e.g. memory loss, particularly in the elderly), aggravate tardive dyskinesia and precipitate psychiatric symptoms (Cookson et al, 2002). The current consensus is that anticholinergic drugs should be administered only to patients who are experiencing extrapyramidal symptoms. However, the majority of patients on therapeutic doses of typical antipsychotics do not experience gross neurological side-effects.

Another significant finding was the high rate of p.r.n. prescribing, which was associated with high doses and polypharmacy, and should be discouraged. A Cochrane systematic review of randomised trials comparing 'as required' medication regimens with regular, fixed, non-discretionary regimens for schizophrenia found no evidence to support the common practice of p.r.n. prescription (Chakrabrati et al, 2007). The other objection is that the attending nurse may not have the requisite expertise to decide on the appropriateness and safety of extra doses. Thus, p.r.n. prescribing may be appropriate for unpredictable patients but, in terms of safety, it is good practice for the prescriber to specify indications for each p.r.n. dose, for example 'one hour delay in falling asleep', or to restrict the maximum number of doses within a given period, for example 'not more than 3 p.r.n. doses in 24 hours'.

Tranquillisation with parenteral antipsychotics should be avoided, although this is rather impracticable in a lowincome country, due to staff shortages and high case-loads. Relating multiple administration to daily schedules (e.g. three times daily) is pharmacologically not justified because most psychotropic drugs have a long biological half-life; furthermore, it consumes considerable nursing time.
A notable feature was the low prescription rate for atypical antipsychotics, despite their efficacy and satisfactory sideeffect profile. The clinicians were apparently aware that the large majority of patients had a low income (the minimum wage in Nigeria is US\$54 per month), hence they were unwilling to prescribe them, but curiously they are comparable in efficacy to some typical antipsychotics (Gureje et al, 2007).

Overall, the pattern of prescribing psychotropic drugs observed was similar to that in several other low- and medium-income countries. Increasing sensitivity to patients' needs had led to a number of ameliorative measures. For instance, education of prescribers by the use of a manual (Baker et al, 2008), routine checks by and feedbacks from pharmacists on adherence to rational drug use and a combination of education and reminders on medication charts (Thompson et al, 2008) have been found to be useful. However, presently in Nigeria, as perhaps elsewhere in subSaharan Africa, such feedback seems impracticable, in that most pharmacists are in private practice and hence have no statutory links with psychiatric hospitals. Alternatively, measures to stress the importance of rational drug use should be in place in psychiatric training curricula and prescribing guides for clinicians.

\section{Acknowledgements}

We thank Dr O. Ihenyen, Medical Director of Psychiatric Hospital, Benin City, Nigeria, for her approval of the study. Her support and the technical inputs of Drs O. C. Ikeji and S. O. Olotu are gratefully appreciated.

\section{References}

Baker, J. A., Lovell, K. \& Harris, N. (2008) The impact of a good practice manual of professional practice associated with psychotropic PRN in acute mental health wards: an exploratory study. International Journal of Nursing Studies, 45, 1403-1410.

Barbul, C. \& Tansella, M. (2005) Psychotropic drug epidemiology: the road travelled, the road ahead. International Review of Psychiatry, 17, 143-145.

Broekema, W. J., de Groot, I. W. \& Van Harten, P. N. (2007) Simultaneous prescribing of atypical antipsychotics, conventional antipsychotics and anticholinergics - a European study. Pharmacy World and Science, 29, 126-130.

Chakrabrati, A., Whicher, E. V., Morrison, M., et al (2007) As required medication regimens for seriously mentally ill people in hospital. Cochrane Database of Systematic Reviews, (3), CD003441.

Cookson, J. C., Taylor, J. \& Katona, D. (2002) Extra pyramidal side-effects. In Use of Drugs in Psychiatry: The Evidence from Psychopharmacology (5th edn), pp. 184-198. Gaskell. 
Edwards, S. \& Kumar, V. (1984) A survey of prescribing of psychotropic drugs in a Birmingham psychiatric hospital. British Journal of Psychiatry, 145, 502-507.

Famuyiwa, O. O. (1988) Psychotropic polypharmacy in Nigeria. The danger can be avoided and cost reduced. Tropical Doctor, 18, 7-11.

Gross, F. (1981) Drug utilisation therapy and practice. The present situation in Federal Republic of Germany. European Journal of Clinical Pharmacology, 19, 387-394.

Gureje, O., Chisholm, D., Kola, L., et al (2007) Cost-effectiveness of an essential mental health intervention package in Nigeria. World Psychiatry, 6, 42-48
Thompson, A., Sullivan, S. A., Barley, M., et al (2008) The DEBIT Trial: an intervention to reduce antipsychotic polypharmacy prescribing in adult psychiatric wards - a cluster randomized controlled trial. Psychological Medicine, 38, 705-715.

Xiang, Y.-T., Weng, Y.-Z., Leung, C-M., et al (2007a) Clinical and social determinants of use of depot antipsychotics for patients with schizophrenia in Hong Kong. Hong Kong Journal of Psychiatry, 16, 71-75.

Xiang, Y.-T., Weng, Y.-Z., Leung, C-M., et al (2007b) Exploring the clinical and social determinants of prescribing anticholinergic medication for Chinese patients with schizophrenia. Human Psychopharmacology, $22,173-180$

\section{State of indigenous mental health in Australia - a colonial legacy?}

\section{Sanil Rege MBBS MRCPsych FRANZCP}

Consultant Psychiatrist, Northern Sydney Central Coast Mental Health Service, Australia, email rege_1@yahoo.es

$$
T_{\text {che }}^{\text {he }}
$$
he Aboriginal culture of Australia is one of the oldest cultures on earth, dating back 50000 years. The Aboriginal and Torres Strait Islander (ATSI) people are the indigenous inhabitants of Australia, constituting $2.4 \%$ of the population. The health status of these 'First Australians' has been described as a source of national shame, with the life expectancy approximately 17 years lower than that of other Australians (Eades, 2000). This gap in life expectancy is also significantly larger than that of other countries with indigenous populations and a history of colonisation, such as the USA, Canada and New Zealand (Ring \& Firman, 1998). This paper offers an overview of the impact of colonisation and its subsequent influence on the social and emotional well-being of ATSI people.

\section{Culture and history}

Colonial domination ... is made possible by the negation of national reality, by new legal relations introduced by the occupying power, by the banishment of the natives and their customs to outlying districts by colonial society, by expropriation, and by the systematic enslaving of men and women. (Fanon, 1963)

Aboriginal mental health cannot be separated from its historical context. Australia was colonised by the British in 1788. At this time there were 300000 Aborigines with 250 distinct languages. Aboriginal society was collectivistic, with extended family clans and traditional kinship structures governed by a set of social laws, customs, rituals and cultural beliefs which differed from one clan to the other. Land was central to the concept of the Aboriginal self. The advent of the British led to the declaration of Australia as 'Terra Nullius' (no man's land), followed by several years of conflict, marginalisation and forced acculturation, with resultant loss of traditional society, religion, law, land and cultural identity. One of the most detrimental policies was the forcible removal of 'half-caste' Aboriginal children from their families and sending them away to work for non-indigenous people with the aim that they would, over time, 'merge' with the non-indigenous population. This began in the 19th century and became public policy in the 20th century; it was still in place as late as 1970. Many hundreds of thousands of children suffered psychological distress through loss of family and culture, physical and sexual abuse, exploitation and institutionalism (HEREOC, 1997). The catastrophic effects of this policy have been highlighted in the Bringing Them Home report, some of which include identity issues, lack of trust and intimacy, delinquency, high rates of depression and suicide, and poor parenting and relationships, thus establishing a vicious circle, producing the 'stolen generations'. This policy, which has been described as genocide (Totten et al, 2007), continues to exert its influence on the psychological well-being of Aboriginal people through intergenerational transmission. It was only in 1967 that Aboriginals were afforded the status of citizens of Australia; previously they came under the Flora and Fauna Act.

Aboriginals continue to maintain strong links to their traditional culture and lands. Data from the 2002 National Aboriginal and Torres Strait Islander Social Survey revealed that $54 \%$ identified with a clan or tribal group, $22 \%$ currently lived on traditional lands, $68 \%$ had participated in cultural events in last 12 months and 14\% did not speak English as their primary language (see http://abs.gov.au/ausstats/abs@.nsf/cat/4714.0).

\section{Social and emotional well-being of Aboriginal Australians}

Aboriginal concepts of mental health are holistic, as illustrated in the following quotation from the Ways Forward report on ATSI mental health:

Health does not just mean the physical well-being of the individual but refers to the social, emotional and cultural well-being of the whole community. This is a whole of life view and includes the cyclical concept of life-death-life. (Swan \& Raphael, 1995) 